\title{
PERCEPÇÃO DE SAÚdE POR SEXO DOS TRABALHADORES NO BRASIL
}

PERCEPTION OF HEALTH BY SEX OF WORKERS IN BRAZIL

Bruna Naiara de Castro ${ }^{1}$

Jefferson Andronio Ramundo Staduto ${ }^{2}$

Fernanda Cristina Ferro Malacoski ${ }^{3}$

\section{RESUMO}

As pesquisas sobre percepção de saúde são relevantes para contribuir com a avaliação de bemestar dos países, uma vez que o estado de saúde afeta diretamente a produtividade do trabalho e renda dos indivíduos, bem como sua qualidade vida. As desigualdades de saúde entre os sexos afetam de forma desproporcional homens e mulheres. O objetivo desta pesquisa consiste em analisar os fatores que influenciam a percepção de saúde de homens e mulheres. Para isso, foram utilizados dados transversais para fazer as análises descritivas e estimar os modelos logit ordinal para o Brasil e por sexo dos trabalhadores. A base de dados utilizada foi a Pesquisa Nacional de Saúde (2013). Os principais resultados mostraram que em média as mulheres declararam menor percepção de boa saúde do que os homens, além disso, independe da faixa salarial, as chances das mulheres declararem estado de saúde muito bom eram menores em relação aos homens.

Palavras-chave: percepção, saúde, gênero, desigualdade.

\begin{abstract}
Research on health perception is relevant to the development of nations, since health status directly affects the productivity and income of individuals; in turn, gender inequalities disproportionately affect the health of both sexes. The objective of this research is to analyze the factors that influence the health perception of men and women. For this, cross-sectional data were used to perform descriptive analyzes and estimate logit ordinal models for Brazil and gender of workers. The database used was the Pesquisa Nacional de Saúde (2013). The main results showed that on average women reported a lower perception of good health than men, moreover, independent of the salary range the chances of women declare very good health were lower compared to men.
\end{abstract}

Key words: perception, health, gender, inequality.

Classificação JEL: I00, I20, I30.

\section{INTRODUÇÃO}

Durante muito tempo os estudos sobre saúde utilizaram indicadores de mortalidade. Contudo, o aumento da longevidade trouxe a necessidade de elaboração de novos indicadores de saúde que incluíssem medidas de qualidade de vida. Muitos estudos sobre bem-estar, mais

1 Mestre em economia pela Universidade Estadual do Oeste do Paraná (UNIOESTE), bn_castro@hotmail.com

2 Doutor em Economia Aplicada pela Universidade de São Paulo (USP), professor da Universidade Estadual do Oeste do Paraná (UNIOESTE) - Toledo. Bolsista produtividade pelo CNPq, jefferson.staduto@unioeste.br

3 Doutoranda em administração pela Universidade de Maringá (UEM), fer-crisferro@hotmail.com 
recentemente, vêm adotando a abordagem multidimensional em substituição à perspectiva unidimensional associado à renda e ao consumo, e a saúde é uma dessas dimensões. Ao longo das últimas décadas, a percepção de saúde vem sendo amplamente utilizada para descrever o estado de saúde da população, pois uma vida longa não significa necessariamente uma vida saudável (SZWARCWALD et al., 2015). Os estudos de Dachs (2002), Maia e Rodrigues (2010), Hosseinpoor et al. (2012), Singh et al. (2013), Soytas e Kose (2014) utilizaram a percepção de saúde autodeclarada como medida do estado de saúde dos indivíduos.

No tema sobre a saúde dos trabalhadores, um dos aspectos que chama a atenção são as diferenças existentes entre homens e mulheres. As desigualdades em saúde podem ser interpretadas como resultado do acesso desigual às várias políticas públicas, tais como previdência, saúde e créditos por indivíduos que pertencem ao mesmo grupo social. Já a equidade em saúde pode ser interpretada como o resultado de políticas que atendam às necessidades específicas de indivíduos que não são iguais (VIEIRA-DA-SILVA; ALMEIDAFILHO, 2009). O sexo distingue os diferentes papéis socialmente construídos, normas, comportamentos, atividades e atributos que determinada sociedade considera apropriados para homens e mulheres, portanto, as desigualdades de gênero afetam de forma desproporcional a saúde das mulheres (HOSSEINPOOR et al. 2012; SOYTAS; KOSE, 2014; FERNANDES et al., 2012). Por sua vez, essas mulheres são heterogêneas e sobre elas pesam os marcadores sociais da diferença de gênero, tais como, sexualidade, idade/geração, raça/cor, classe social e corporalidades que interagem contextual e conjunturalmente, de modo a promover potenciais cenários de desigualdades sociais e hierarquizações, o que revela a grande interseccionalidade de gênero na saúde das mulheres.

Proteger a saúde de homens e mulheres não é apenas um direito humano básico, mas, também, crucial para o desenvolvimento econômico e social da nação e para atingir níveis mais altos de bem-estar. Compreender a desigualdade de percepção de saúde dos trabalhadores é importante para a formulação de políticas públicas de saúde e, se o sexo influencia na percepção de saúde, as políticas de saúde específicas ao sexo podem ajudar na redução dessa desigualdade de gênero (SOYTAS; KOSE, 2014). A distinção é também necessária porque o comportamento de ambos os sexos é diferente, e isso reflete na utilização de serviços de saúde e também no estado de saúde do indivíduo (BARATA, 2012). Além disso, a perspectiva de gênero na análise da saúde deve ser adotada porque as mulheres são em maior número, vivem mais tempo, e estão mais expostas à pobreza e à precariedade (FERNANDES et al., 2012). Neste contexto, o objetivo desta pesquisa consiste em analisar os fatores que influenciam a percepção de saúde de trabalhadores por sexo no Brasil.

\section{MERCADO DE TRABALHO E SAÚDE}

A saúde dos trabalhadores é uma parte do capital humano porque é essencial para aumentar a produtividade e, consequentemente, os rendimentos (SCHULTZ, 1973; BECKER, 1994). Schultz (1973) argumenta que o capital humano não é composto apenas por educação formal e treinamento, mas, também, pela saúde dos trabalhadores, isto é, todo o gasto que afeta a expectativa de vida, a capacidade de resistência, o vigor físico e a vitalidade de um povo. $\mathrm{O}$ capital humano é de extrema importância para determinar o crescimento e o desenvolvimento econômico da economia. Alguns economistas empenharam-se em determinar os efeitos dos progressos verificados no campo da saúde por meio da mensuração da saúde, pois esses incrementam a qualidade dos recursos humanos, por exemplo, a alimentação adicional e condições de moradia. Para Becker (1994), um alto nível de saúde da população reduz a taxa 
de mortalidade e induz um aumento nos investimentos em educação e outros tipos de capital humano, uma vez que a taxa de retorno desses investimentos será maior quanto mais tempo de vida tiver o indivíduo. O investimento em capital humano é uma das maneiras mais eficientes de aumentar o nível de renda e de saúde da população mais pobre.

A família é um importante componente na composição do capital humano. A renda da família influencia os ganhos futuros dos filhos. Pais mais educados e com mais cultura formal têm melhores condições de ajudar no desenvolvimento das capacidades, da cultura formal, da educação e das habilidades de seus filhos. De outro lado, os pais que têm pelo menos o nível básico de instrução, elaboram um planejamento, a fim de direcionar os filhos a receber um melhor nível de educação. Trata-se de uma função de utilidade, em que os pais abrem mão do consumo de bens e serviços, porque acreditam no retorno da educação futura dos filhos. Os pais, neste caso, são investidores, já que estão renunciando a alguns gastos, reduzindo seu consumo para gastar com habilidades, saúde, aprendizado e motivação para seus filhos (BECKER, 1962).

Além disso, a saúde tem impacto sobre a oferta de trabalho, mais especificamente na escolha setorial, na decisão de trabalhar e no número de horas a ser ofertado. Considerando as duas últimas questões, o resultado de uma saúde precária sobre a oferta de trabalho é ambíguo, dependendo da combinação de três efeitos. O primeiro "efeito é o da dotação", pelo qual o indivíduo doente dispõe de menos tempo para realizar suas atividades normais, inclusive para o trabalho. O segundo "efeito é o da substituição", assim, se uma saúde precária corresponde a uma taxa de salários mais baixa, o trabalhador decide reduzir a sua oferta de trabalho, já que esse trabalho gera uma desutilidade ao indivíduo, portanto, há um efeito de substituição entre trabalho e lazer. Por fim, o terceiro "efeito é o da renda", que faz o doente trabalhar mais para compensar os menores rendimentos por hora. Se o efeito renda for grande o suficiente para compensar o efeito dotação mais o efeito substituição, uma saúde precária resulta em maior oferta de trabalho. Caso contrário, um indivíduo doente tende a trabalhar menos tempo do que uma pessoa saudável (STRAUSS, 1998; ALVES; ANDRADE, 2003).

O stress no local de trabalho desempenha papel importante nas diferenças de saúde. Existe um círculo virtuoso entre saúde e produtividade, pois melhorias nas condições de trabalho levará a uma força de trabalho mais saudável, o que levará a uma melhor produtividade e, consequentemente, a uma oportunidade de criar um ambiente de trabalho ainda mais saudável e mais produtivo (WILKINSON; MARMOT, 2003). Os problemas de saúde do trabalhador estão relacionados a uma menor produtividade e desempenho, por outro lado, com o aumento de acidentes e ausência ou licença do trabalho (MÄKINEN; HASSI, 2009). Por isso, as empresas envolvem-se com atividades de promoção de saúde e esperam a diminuição do absenteísmo e do custo da assistência médica. Ademais, a promoção da saúde representa uma imagem positiva da empresa tanto internamente quanto para a sociedade (SILVA, 2004).

Com um nível de saúde precário dos trabalhadores todos perdem no processo produtivo no conjunto da firma, desde o operacional aos extratos mais altos da hierarquia. Com investimento realizado em saúde, os funcionários tendem a reduzir a prevalência de doenças, portanto, reduz o absenteísmo por motivo de saúde. Dessa forma, as empresas podem aumentar a lucratividade em função de os trabalhadores poderem expressar toda a capacidade laboral, por outro lado, há redução de despesas com o afastamento por razões de saúde (SCHIEHL et al., 2012).

As empresas buscam aumentar a sua produtividade por meio de avanços tecnológicos, por outro lado, esses avanços são operados pelos trabalhadores. Dessa forma, as empresas devem investir no homem por meio da promoção do bem-estar, da saúde e da qualidade de vida, 
buscando como resultado pessoas mais produtivas (SGANZERLA, 2007). Os trabalhadores com fatores de riscos ou problemas de saúde acabam por comprometer a produtividade e geram um custo maior para a empresa relacionado à sua ausência no trabalho, resultando em sobrecarga nos demais trabalhadores (SCHIEHL et al., 2012).

O estado de saúde ruim exclui do mercado de trabalho algumas pessoas, principalmente adultos e idosos. Os efeitos da exclusão para os idosos são os mais proeminentes, pois, nessa idade, existe maior proporção de doentes, com doenças mais graves que, por sua vez, geram inaptidão física ou cognitiva (NORONHA, 2005).

Do ponto de vista dos fatores ocupacionais que podem determinar a percepção do estado de saúde por parte dos trabalhadores, Ferreira e Santana (2003) afirmam que trabalhadores manuais apresentam uma tendência mais negativa de autoavaliação de saúde do que aqueles que trabalham com atividades que não são manuais. Inferem que o tipo de ocupação manual age negativamente sobre a percepção de saúde do indivíduo.

Stiglitz, Sen e Fitoussi (2009) destacaram que alguns padrões foram encontrados em pesquisas sobre a diferença de estado de saúde. As pessoas inseridas em classes ocupacionais mais baixas, com menor educação formal e com menor renda, estão propensas a morrerem mais jovens e a apresentarem maior incidência de problemas de saúde.

O trabalhador que se encontra em uma pior condição de saúde percebe que essa situação afeta a sua predileção por determinado trabalho, a chance de auferir determinado salário, o tempo de trabalho e a disposição em se aposentar (DWYER; MITCHELL, 1998). Somase ainda que, de acordo com uma pesquisa realizada com russos, as pessoas que receberam menores rendimentos apresentaram pior saúde e maior ocorrência de desemprego (STIGLITZ; SEN; FITOUSSI, 2009).

O status de saúde como "pobre" afeta a produtividade, diminui os ganhos e influencia as escolhas, por exemplo, entre consumo e lazer, já que o trabalho se torna mais árduo e menos gratificante. Dessa forma, influencia a escolha pelo lazer ou aposentadoria, diminuindo a quantidade de anos trabalhados e com isso a restrição orçamentária (DWYER; MITCHELL, 1998). A diminuição dos ganhos originários do salário como consequência de um pior nível de saúde influencia o bem-estar individual e de toda a sociedade. Isso resulta em efeitos sobre os indicadores macroeconômicos, como distribuição de renda e riqueza da população (NORONHA, 2005).

\section{DIFERENÇAS DE PERCEPÇÃO DE SAÚDE ENTRE HOMENS E MULHERES}

É necessária a distinção entre os sexos, porque os sujeitos apresentam comportamentos distintos oriundos do contraste entre o masculino e o feminino. O papel da mulher na sociedade já esteve intensamente assentado na reprodução biológica, com ênfase na maternidade e nos afazeres domésticos. Já o papel do homem era definido por meio do exercício dominante do poder econômico e da esfera pública. Essa dicotomia entre os papéis masculino e feminino, embora esteja traçada ao longo dos séculos e consagrada em uma relativa divisão sexual do trabalho, tem mudado bastante na história recente nas culturas ocidentalizadas (KON, 2002; MELO, 2007).

O sexo ganhou importância na análise da equidade e cuidados com a saúde por chamar a atenção para um tipo específico de iniquidade, referente à natureza social, econômica e cultural de cada sexo. É necessário identificar as semelhanças e as diferenças nas necessidades 
de saúde entre homens e mulheres, bem como identificar e analisar os obstáculos que, com base no sexo, os impedem de permanecerem saudáveis. As atitudes dos homens referentes à saúde têm relação com as suas vivências, o que é resultado dos padrões de masculinidade. As diferenças biológicas entre homens e mulheres foram durante muito tempo consideradas como um dos principais fatores que inviabilizavam a comparação entre a saúde de homens e mulheres (FERNANDES et al., 2012).

A organização dos cuidados e dos serviços de saúde deveria estar de acordo com as necessidades claramente distintas. Essa diferença relativa ao sexo não se limita à saúde reprodutiva, mas, também, incluíam diferenças genéticas, hormonais e metabólicas consideradas determinantes dos padrões de mortalidade e morbidade (DOYAL, 2001). Além das diferenças biológicas, outros aspectos englobam a problemática da desigualdade de saúde. As mulheres apresentarem percepções inferiores de saúde, independente de outras variáveis, estaria diretamente relacionada às desigualdades de gênero reproduzidas na sociedade. Essa constatação sugere o efeito independente do sexo sobre o estado de saúde (FERREIRA; SANTANA, 2003). Nesse sentido, os diferenciais em termos de participação na força de trabalho, independência financeira e responsabilidades domésticas contribuem para as diferenças de percepção de saúde de sexo ao longo da vida (PRUS; GEE, 2003).

Existem duas hipóteses principais sobre mecanismos sociais que poderiam explicar as diferenças de sexo nos resultados de saúde: i) diferencial de exposição sugere que as mulheres relatam níveis de saúde mais baixos do que os homens devido às maiores exigências e obrigações em seus papéis sociais e níveis mais baixos de recursos para ajudá-las a lidar com essas condições. Isto implica que equivalentes papéis sociais e recursos iguais devem eliminar as diferenças de sexo nos resultados de saúde; ii) diferencial de vulnerabilidade faz referência a maior capacidade de resposta das mulheres aos eventos da vida e tensões contínuas que são experimentadas igualmente por ambos os sexos. No entanto, nenhuma das duas hipóteses encontram pleno apoio empírico (SOYTAS; KOSE, 2014).

Além disso, é relevante destacar a interseccionalidade, ou seja, as articulações entre a discriminação de gênero, a homofobia, o racismo e a exploração de classe, bem como outros marcadores sociais. Esse conceito revela sistemas múltiplos e interseção de opressão e privilégio (HANKIVSKY; CHRISTOFFERSEN, 2008; TAQUETTE, 2010). No âmbito da saúde, a abordagem da interseccionalidade tem potencial para fornecer novos caminhos e orientar ações de forma mais eficaz com intuito de eliminar as disparidades de saúde nas diversas dimensões críticas da desigualdade social (WEBER; PARRA-MEDINA, 2003).

Essas relações desiguais atravessam todas as dimensões da vida social, têm uma dinâmica própria, independentemente de outros processos sociais, e são marcadas pelo antagonismo, isto é, pela relação hierárquica dos homens sobre as mulheres. As mulheres, consequentemente, acabam inseridas em espaços desvalorizados sócio e economicamente. Destaca-se que as características do trabalho feminino podem levar ao desgaste físico, psicológico e, assim, interferir na forma como as mulheres avaliam sua saúde (SINGH et al., 2013; BARATA, 2012, SOYTAS; KOSE, 2014). A equidade na saúde decorre do reconhecimento de que algumas das diferenças existentes são expressões das desigualdades sociais. A principal razão para estudos de saúde sensíveis ao gênero é providenciar informações para definir planos de políticas e ações que possam reduzir essas inequidades e melhorar os resultados em termos de saúde (FERNANDES et al., 2012). 


\section{METODOLOGIA}

A base de dados utilizada nessa pesquisa refere-se à Pesquisa Nacional de Saúde de 2013 (PNS) do Instituto Brasileiro de Geografia e Estatística (IBGE). A variável de interesse principal refere-se à percepção de saúde do indivíduo. A pergunta que se fez é: "De um modo geral, como é o estado de saúde?" Com cinco categorias de respostas: "muito ruim", "ruim", "regular", "bom" e "muito bom". O indicador de percepção de saúde autodeclarada tem a vantagem de ser uma medida ampla do estado de saúde, que engloba todas as morbidades, e tem relação com a mortalidade da população, contemplando para além do bem-estar físico, como o social e o mental, e constituindo-se em um indicador multidimensional da saúde (IDLER; BENYAMINI, 1997; BENJAMIN et al., 2004).

É importante considerar alguns problemas relacionados à percepção de saúde nas pesquisas domiciliares: a percepção de saúde pode ser diferente entre distintos grupos sociais, étnicos ou culturais. Outro problema se refere ao informante das respostas, pois não é possível garantir a presença de todos os moradores do domicílio no momento da entrevista, sendo que a avaliação daqueles ausentes acaba sendo subjetivamente feita por outra pessoa (JÜRGES, 2007; MAIA; RODRIGUES, 2010).

Nas pesquisas sobre percepção de saúde, tanto no âmbito nacional como internacional, geralmente a variável foi codificada em 1 (muito ruim), 2 (ruim), 3 (regular), 4 (bom), 5 (muito bom). Portanto, a variável dependente apresenta-se em escalas ordinais, uma vez que está clara a hierarquia entre as categorias. Nesta pesquisa, o modelo utilizado corresponde ao Logit ordinal, que de acordo com Wooldridge, (2010), pode ser representado na forma estrutural:

Em que é uma variável ordenada que assume os valores $\{1,2,3,4,5\}$; =é um vetor cujos elementos correspondem aos efeitos das variáveis explicativas na variável resposta; = as variáveis explicativas; = termo de erro estocástico. O modelo não apresenta constante, mas pontos de corte (cut points). Na equação (1) do logit ordinal, mede a variação em para uma unidade de variação em, ou seja, nesta pesquisa indica quanto o logaritmo das chances do indivíduo declarar-se com estado de saúde muito bom varia em resposta à mudança de uma unidade da variável explicativa. Calculou-se a variação no odds ratio por meio do antilogaritmo de , ou seja, calcular $e^{\beta}$. A Tabela 1 apresenta as "descrições", "média", "desvio padrão", "mínimo" e "máximo" da variável dependente e das variáveis explicativas usadas nos modelos logit ordinal para homens e mulheres.

\section{TABELA 1 - DESCRIÇÃO, MÉDIA, DESVIO PADRÃO E MÍNIMO E MÁXIMO DAS VARIÁVEIS UTILIZADAS PARA HOMENS E MULHERES - BRASIL}

\begin{tabular}{|c|c|c|c|c|c|c|c|}
\hline \multirow[b]{2}{*}{ Variáveis } & \multirow[b]{2}{*}{ Descrição } & \multicolumn{2}{|c|}{ Homens } & \multicolumn{2}{|c|}{ Mulheres } & \multirow[b]{2}{*}{ Min. } & \multirow[b]{2}{*}{ Max. } \\
\hline & & Média & $\begin{array}{l}\text { Desvio } \\
\text { Padrão }\end{array}$ & Média & $\begin{array}{l}\text { Desvio } \\
\text { Padrão }\end{array}$ & & \\
\hline Percepção de saúde & $\begin{array}{l}\text { Muito Ruim=1; Ruim=2; Regu- } \\
\text { lar=3; Bom=4; Muito Bom=5. }\end{array}$ & 3,88 & 0,67 & 3,84 & 0,70 & 1 & 5 \\
\hline Idade & Anos de idade do ocupado. & 37,85 & 12,16 & 37,58 & 11,58 & 18 & 65 \\
\hline Branca & Branca $=1 ;$ Não Branca $=0$. & 0,39 & 0,49 & 0,43 & 0,49 & 0 & 1 \\
\hline Urbano & Urbano=1; Rural=0. & 0,82 & 0,38 & 0,89 & 0,31 & 0 & 1 \\
\hline Pobreza & $\begin{array}{l}\text { Variável binária para rendimen- } \\
\text { to domiciliar per capita menor } \\
\text { que } 1 / 2 \text { salário mínimo. }\end{array}$ & 0,26 & 0,44 & 0,20 & 0,40 & 0 & 1 \\
\hline
\end{tabular}


Nível de Escolari-

dade

Fundamental in-

completo

Fundamental com-

pleto

Médio completo

Superior completo

Jornada de trabalho

Até 35 horas por

semana

De 36 a 44 horas

por semana

Mais de 45 horas

por semana

Número de filhos

Variável binária para jornada de

Variável binária para cada nível de escolaridade. Sendo o nível Fundamental incompleto usado como referência.

$\begin{array}{llllll}0,35 & 0,48 & 0,23 & 0,42 & 0 & 1 \\ 0,17 & 0,37 & 0,14 & 0,35 & 0 & 1 \\ 0,36 & 0,48 & 0,42 & 0,49 & 0 & 1 \\ 0,12 & 0,33 & 0,21 & 0,41 & 0 & 1\end{array}$

trabalho tendo escolhido como referência a jornada de trabalho

$\begin{array}{llllll}0,15 & 0,36 & 0,31 & 0,46 & 0 & 1 \\ 0,54 & 0,50 & 0,50 & 0,50 & 0 & 1\end{array}$

de 36 a 44 horas por semana.

Quantas vezes con-

sultou o médico

Número de filhos que nasceram vivos.

$\begin{array}{llllll}0,54 & 0,50 & 0,50 & 0,50 & 0 & 1\end{array}$

$\begin{array}{llllll}0,31 & 0,46 & 0,19 & 0,39 & 0 & 1\end{array}$

$\begin{array}{llllll}(\ldots) & (\ldots) & 0,53 & 1,15 & 0 & 22\end{array}$

Número de vezes que consultou o médico nos últimos 12 meses.

$\begin{array}{llllll}2,83 & 3,21 & 3,90 & 4,21 & 1 & 60\end{array}$

Diagnosticado

(doença crônica)

Diagnosticado=1; Não Diagnosticado $=0$.

$\begin{array}{llllll}0,10 & 0,30 & 0,14 & 0,35 & 0 & 1\end{array}$

Plano de saúde=1; Não possuía Plano de Saúde $=0$.

$\begin{array}{llllll}0,27 & 0,44 & 0,35 & 0,48 & 0 & 1\end{array}$

Regiões do País

Sul

Nordeste

Norte

Centro-Oeste

Sudeste

\begin{tabular}{lllllll} 
& 0,12 & 0,33 & 0,13 & 0,34 & 0 & 1 \\
Quatro variáveis binárias para & 0,29 & 0,45 & 0,28 & 0,45 & 0 & 1 \\
distinguir cinco regiões, a região & 0,23 & 0,42 & 0,20 & 0,40 & 0 & 1 \\
do Sudeste foi a referência. & 0,13 & 0,33 & 0,13 & 0,34 & 0 & 1 \\
& 0,23 & 0,42 & 0,25 & 0,44 & 0 & 1 \\
\hline
\end{tabular}

Fonte: Elaboração própria, com base nos microdados da Pesquisa Nacional de Saúde de 2013 - PNS.

Nota: (...): informação em falta.

As estimavas foram realizadas para homens e mulheres ocupados, a amostra selecionada contempla 56.183 indivíduos com idade entre 18 e 65 anos. Tanto as estatísticas descritivas como os resultados das estimativas levam em consideração o fator de expansão para dados complexos da PNS 2013, assim os resultados demonstrados são representativos para a população.

\section{RESULTADOS E DISCUSSÕES}

Na Tabela 2, observa-se que a maioria, tanto dos homens como das mulheres, declarou ter estado de saúde "bom". Contudo, as mulheres apresentaram maiores percentuais nos estratos "regular", "ruim" e "muito ruim". Não há um consenso sobre os mecanismos que levam às diferenças de sexo nos resultados de saúde; a pior percepção de saúde das mulheres está relacionada às diferenças biológicas, sociais, dupla jornada de trabalho, além da inserção precária no mercado de trabalho (SOYTAS; KOSE, 2014). 
TABELA 2 - PERCEPÇÃO DE SAÚDE DECLARADA POR SEXO - BRASIL

\begin{tabular}{ccc}
\hline Percepção de Saúde & Mulher (\%) & Homem (\%) \\
\hline Muito Ruim & 0,62 & 0,39 \\
Ruim & 2,50 & 2,15 \\
Regular & 22,88 & 19,29 \\
Bom & 59,59 & 62,14 \\
Muito Bom & 14,42 & 16,03 \\
\hline Total & 100 & 100 \\
\hline
\end{tabular}

Fonte: Elaboração própria, com base nos microdados da Pesquisa Nacional de Saúde de 2013 - PNS.

Ross e Wu (1995) argumentam que o efeito da escolaridade é relevante na percepção de saúde por dois aspectos principais: o nível de instrução é a principal causa das desigualdades sociais em saúde porque se reflete nos rendimentos dos indivíduos; e indivíduos com maiores níveis de escolaridade são mais propensos a adotar hábitos de vida saudáveis, tais como a prática de atividade física, a manutenção de peso adequado, o consumo moderado de álcool e o hábito de não fumar. Para homens e mulheres, quanto maior o nível de instrução, maior o percentual de indivíduos que declararam estado de saúde "muito bom" (Gráfico 1). Para o estado de saúde declarado como "bom", observa-se para ambos os sexos o formato de U invertido, ou seja, o percentual aumenta inicialmente com níveis de instrução mais altos e depois passa a diminuir.

\section{GRÁFICO 1 - PERCEPÇÃo DE SAÚDE DECLARADA POR SEXO E NÍVEL DE INSTRUÇÃO - BRASIL}

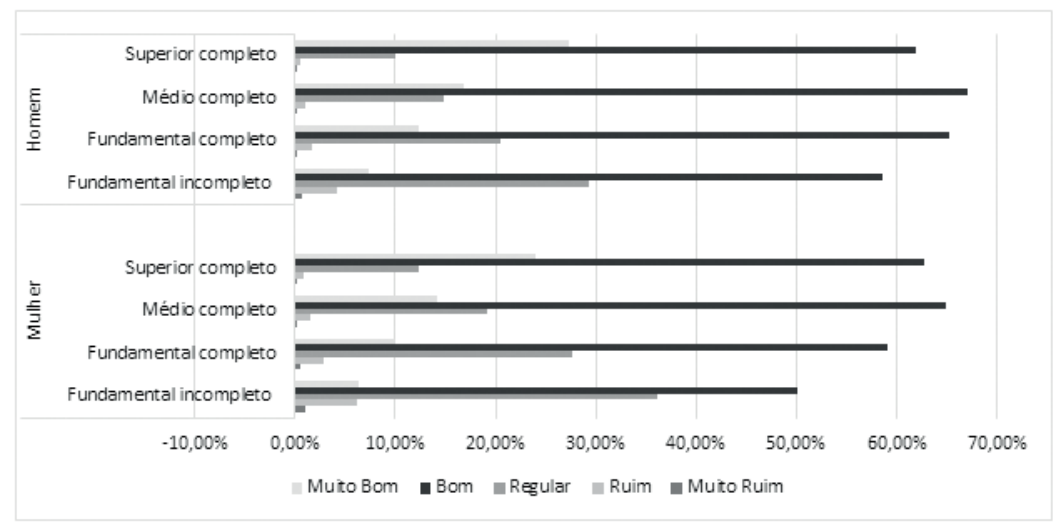

Fonte: Elaboração própria, com base nos microdados da Pesquisa Nacional de Saúde de 2013 - PNS.

Entre os determinantes de saúde, as condições socioeconômicas são as que melhor explicam as diferenças de morbidade e mortalidade no final da vida (FERNANDES et al., 2012). Para o Brasil, os resultados apresentados no Gráfico 2 confirmam a relação positiva entre estado de saúde declarado como "muito bom" e salário, pois à medida que aumenta a faixa de salário, também aumentam os percentuais de homens e mulheres mais saudáveis. O percentual de mulheres que declararam estado de saúde "muito bom" é superior ao dos homens em todas as faixas de salários, exceto na faixa $>5 \mathrm{e} \leq 10$. Nesse sentido, em Portugal, Vintém, Guerreiro e Carvalho (2004) observaram que as desigualdades de saúde por sexo nas classes sociais de baixa renda têm a tendência de declarar negativamente seu estado de saúde e descreveram sentimento positivo de bem-estar menos frequente, além disso, declararam que 
vão com mais frequência às consultas médicas. O estudo de Deaton (2003) mostrou que a renda é mais relevante principalmente na promoção da saúde dos mais pobres.

\section{GRÁFICO 2 - PERCEPÇÃO DE SAÚdE DECLARADA POR SEXO E SALÁRIO PRINCIPAL EM SALÁRIOS MÍNIMOS - BRASIL}

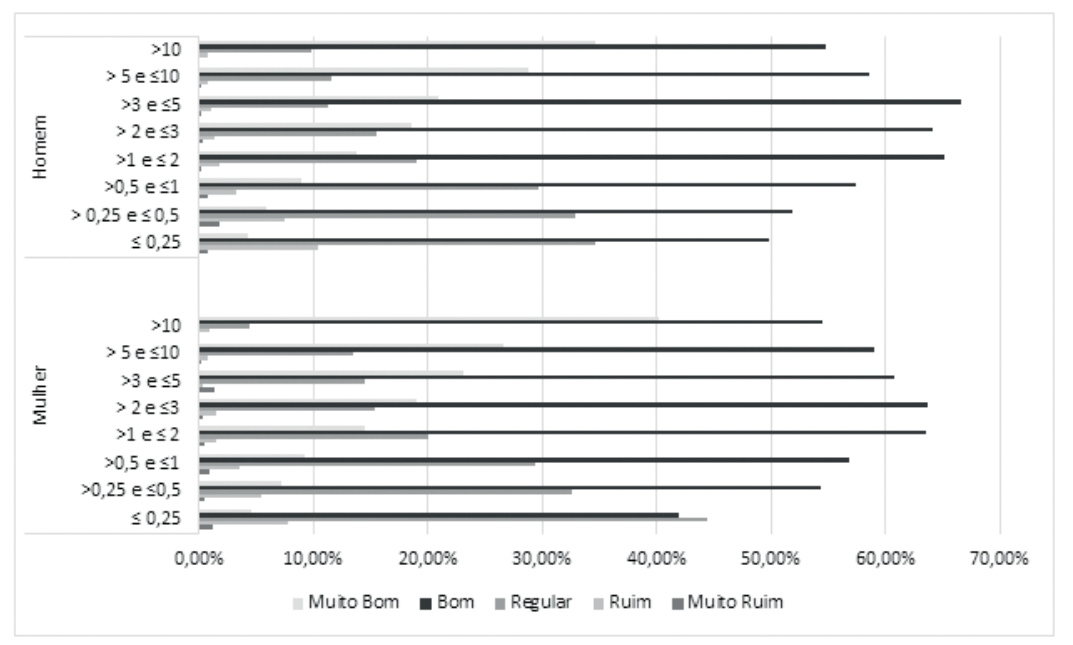

Fonte: Elaboração própria, com base nos microdados da Pesquisa Nacional de Saúde de 2013 - PNS.

$\mathrm{Na}$ Tabela 3, são apresentados os resultados das razões de chances (odds ratio) para o modelo logit ordinal estimado para o Brasil, homens e mulheres. Todos os parâmetros foram significativos, com exceção da jornada de trabalho até 35 horas e residir na Região Sul para os o Brasil e para homens. Dentre os parâmetros estimados para as mulheres, não foi significativo apenas residir na Região Sul. O teste qui-quadrado da razão de verossimilhança - LR chi2 - que avalia a significância conjunta das variáveis foi estatisticamente significativo.

No modelo para o Brasil, a variável mulher apresentou relação negativa com o estado de saúde, ou seja, em média as mulheres apresentam menos chances que os homens de declararem estado de saúde "muito bom". Dessa forma, foram estimados modelos separados por sexo com o intuito de captar o efeito das variáveis selecionadas sobre a saúde de ambos os sexos.

Os resultados mostram que o aumento da "Idade" reduzia as chances de homens e mulheres declararem estado de saúde "muito bom". Esse comportamento revela que o avanço da idade reduz o estoque do capital saúde (GROSSMAN, 1972). Foi observado na Turquia e nos Estados Unidos (SINGH et al., 2013) que o aumento da idade reduz a probabilidade de as mulheres se declararem saudáveis.

Ao longo do processo de envelhecimento acentuam-se as diferenças, as vantagens e desvantagens decorrentes de status socioeconômicos diferentes. A trajetória de vida apresenta altos e baixos resultantes de realizações pessoais e/ou de frustações. Após certa idade, as condições sociais, econômicas e culturais adquiridas condicionam ou contribuem para práticas de cuidados de saúde e para o nível de saúde que o indivíduo irá usufruir na velhice. Assim, o indivíduo em idade avançada depende do capital de saúde construído ao longo de uma vida (VIEIRA-DA-SILVA; ALMEIDA-FILHO, 2009).

Para ambos os sexos, residir na zona "Urbana" e declarar ser da cor "Branca" aumentava as chances de declararem estado de saúde "muito bom". Noronha (2005) mostrou que a grande dispersão geográfica dos moradores das áreas rurais e, por outro lado, a baixa oferta de serviços de saúde pública e privada pode afetar a saúde dessa população. As atividades laborais também 
tendem a ser mais intensas, com jornadas mais longas. Resultados similares foram encontrados em outros estudos (DACHS, 2002; SOUSA, 2010), nos quais indivíduos que declararam ser da cor preta ou parda tinham mais chances de reportarem estado de saúde ruim, essa diferença de percepção negativa em relação à cor não branca era mais intensa para as mulheres do que para os homens.

Os pesquisadores por muitos anos assumem que há uma correlação positiva entre renda e saúde. Entretanto, para os níveis de renda maiores essas evidências diminuem (FUCHS, 2004). A variável "Pobreza" capta o efeito do rendimento domiciliar per capita em salários mínimos. Foram considerados pobres os indivíduos que pertenciam às famílias que recebiam até R\$339,00 per capita, ou seja, 1/2 salário mínimo, em 2013. Assim, homens e mulheres em situação de pobreza tinham menos chances de declararem estado de saúde "muito bom". Nesse sentido, na Índia, Singh et al. (2013) evidenciou que os idosos homens e mulheres tinham menos chances de declararem estado de saúde baixo quanto mais alto era o índice de gasto mensal.

O estado de saúde foi positivamente associado aos níveis educacionais mais altos para homens e mulheres em um estudo com 57 países no período de 2002-2004 (HOSSEINPOOR et al., 2012). No Brasil, as investigações do efeito da educação sobre a saúde dos indivíduos mostraram forte relação positiva entre estado de saúde declarado e anos de estudos (COSTA, 2008; SOUSA, 2010; CASTRO, 2017). A variável "Nível de Escolaridade" foi categorizada em quatro níveis de instrução, no qual ter nível de instrução superior ao Fundamental incompleto aumentavam as chances de homens e mulheres declararem estado de saúde "muito bom".

A jornada de trabalho curta involuntária pode estar associada a situações precárias de empregabilidade e condições de insalubridade. De forma oposta, jornadas excessivas de trabalho estão associadas à incidência de acidentes de trabalho, fadiga, problemas cardiovasculares, entre outros (MAIA; RODRIGUES, 2010). Nesse contexto, ter uma jornada de trabalho de "Até 35 horas" diminuía as chances das mulheres declararem estado de saúde "muito bom". Para a jornada de trabalho de "Mais de 45 horas", as chances de homens e mulheres declararem estado de saúde "muito bom" diminuíam em comparação com a jornada de trabalho de 36 a 44 horas.

Os dados da PNS não apresentam informações sobre o número de filhos para o sexo masculino, apenas para o feminino. Quanto maior o "Número de Filhos" nascidos vivos, as chances de as mulheres declararem estado de saúde "muito bom" diminuíam. A associação entre dupla jornada, casamento e filhos em idade pré-escolar dobrou o risco das mulheres apresentarem sintomas psiquiátricos; por outro lado, na ausência ou baixa intensidade da dupla jornada esta relação desaparecia (BARATA, 2012). Portanto, a sobrecarga gerada pelas atividades de produção e reprodução imputam deterioração da saúde das mulheres. Atualmente, apesar do aumento da taxa de atividade feminina nas últimas décadas, o papel de cuidar dos filhos e da casa ainda é atribuído à mulher, reduzindo o tempo disponível para se dedicar à carreira profissional, o que compromete a remuneração recebida. Portanto, essa variável pode estar captando parte do efeito da dupla jornada de trabalho feminina.

O número de consultas ao médico (Quantas vezes consultou o médico) diminuía as chances de homens e mulheres declararem estado de saúde "muito bom". Esta relação negativa entre a percepção de saúde e a consulta médica faz sentido, uma vez que é por meio da consulta médica que os problemas de saúde podem ser identificados. Além disso, as pessoas, em média, tendem a procurar cuidado médico quando já estão doentes e não por prevenção (COSTA, 2008). 
A variável "Diagnosticado" refere-se ao fato de o indivíduo ter recebido diagnóstico de alguma doença crônica ou de longa duração (mais de seis meses). Assim, receber esse tipo de diagnóstico reduzia as chances de homens e mulheres declararem estado de saúde "muito bom". O estudo de Barreto e Figueiredo (2009) mostrou que homens e mulheres com doenças crônicas não transmissíveis apresentavam pior percepção da saúde. O fato de ter "Plano de Saúde" aumentava as chances de homens e mulheres declararem estado de saúde "muito bom".

As diferenças regionais são relevantes para explicar o estado de saúde dos indivíduos (MAIA; RODRIGUES, 2010). A variável "Regiões do País" mostra que morar na região Nordeste, Norte ou Centro-Oeste em comparação com a região Sudeste, diminuía as chances de homens e mulheres declararem estado de saúde "muito bom". As regiões Norte e Nordeste foram as que apresentaram maiores desvantagens. Os estudos de Travassos, Oliveira, Viacava (2006) e Stopa et al. (2017) mostraram que os indivíduos residentes nas regiões Sul e Sudeste apresentavam maiores chances de uso dos serviços públicos de saúde do que os residentes das demais regiões. Por sua vez, o uso do sistema de saúde está associado à sua distribuição geográfica e oferta.

TABELA 3 - RAZÕES DE CHANCES ESTIMADAS PELO LOGIT ORDINAL, PARA ANALISAR OS DETERMINANTES DA PERCEPÇÃO DE SAÚDE “MUITO BOA" DE HOMENS E MULHERES - BRASIL

\begin{tabular}{|c|c|c|c|c|c|c|}
\hline \multirow[b]{2}{*}{ Variáveis } & \multicolumn{2}{|c|}{ Brasil } & \multicolumn{2}{|c|}{ Homem } & \multicolumn{2}{|c|}{ Mulher } \\
\hline & Odds Ratio & Erro Padrão & Odds Ratio & Erro Padrão & Odds Ratio & Erro Padrão \\
\hline Mulher & $0,87^{* * *}$ & 0,02 & NA & NA & NA & NA \\
\hline Homem & 1 & Ref & NA & NA & NA & NA \\
\hline Idade & $0,97^{* * *}$ & 0,00 & $0,97^{* * *}$ & 0,00 & $0,97^{* * *}$ & 0,00 \\
\hline Branco & $1,18^{* * *}$ & 0,02 & $1,13^{* * *}$ & 0,03 & $1,22^{* * *}$ & 0,03 \\
\hline Não Branco & 1 & Ref & 1 & Ref & 1 & Ref \\
\hline Urbano & $1,31^{* * *}$ & 0,04 & $1,33^{* * *}$ & 0,05 & $1,29^{* * *}$ & 0,05 \\
\hline Rural & 1 & Ref & 1 & Ref & 1 & Ref \\
\hline Pobreza & $0,70^{* * *}$ & 0,02 & $0,66^{* * *}$ & 0,02 & $0,74^{* * *}$ & 0,02 \\
\hline Não Pobreza & 1 & Ref & 1 & Ref & 1 & Ref \\
\hline $\begin{array}{l}\text { Até Fundamental incom- } \\
\text { pleto }\end{array}$ & 1 & Ref & 1 & Ref & 1 & Ref \\
\hline Fundamental completo & $1,27^{* * *}$ & 0,04 & $1,27^{* * *}$ & 0,05 & $1,27^{* * *}$ & 0,05 \\
\hline Médio completo & $1,75^{* * *}$ & 0,04 & $1,71^{* * *}$ & 0,06 & $1,83^{* * *}$ & 0,06 \\
\hline Superior completo & $3,03^{* * *}$ & 0,09 & $2,97^{* * *}$ & 0,13 & $3,11^{* * *}$ & 0,13 \\
\hline \multicolumn{7}{|l|}{ Jornada de trabalho } \\
\hline Até 35 horas por semana & 0,97 & 0,02 & 1,02 & 0,04 & $0,94^{* * *}$ & 0,03 \\
\hline $\begin{array}{l}\text { De } 36 \text { a } 44 \text { horas por } \\
\text { semana }\end{array}$ & 1 & Ref & 1 & Ref & 1 & Ref \\
\hline $\begin{array}{l}\text { Mais de } 45 \text { horas por } \\
\text { semana }\end{array}$ & $0,92^{* * *}$ & 0,02 & $0,93^{* * *}$ & 0,03 & $0,92^{* * *}$ & 0,03 \\
\hline Número de filhos & NA & NA & $(\ldots)$ & $(\ldots)$ & $0,98^{* *}$ & 0,01 \\
\hline $\begin{array}{l}\text { Quantas vezes consultou o } \\
\text { médico }\end{array}$ & $0,91^{* * *}$ & 0,00 & $0,90^{* * *}$ & 0,00 & $0,92^{* * *}$ & 0,00 \\
\hline Diagnosticado & $0,27^{* * *}$ & 0,01 & $0,26^{* * *}$ & 0,01 & $0,28^{* * *}$ & 0,01 \\
\hline Não Diagnosticado & 1 & Ref & 1 & Ref & 1 & Ref \\
\hline Plano de Saúde & $1,87^{* * *}$ & 0,04 & $1,90^{* * *}$ & 0,06 & $1,85^{* * *}$ & 0,05 \\
\hline
\end{tabular}




\begin{tabular}{l|c|c|c|c|cc}
\hline $\begin{array}{l}\text { Não possuía Plano de } \\
\text { Saúde }\end{array}$ & 1 & Ref & 1 & Ref & 1 & Ref \\
Regiões do País & & & & & & \\
Sul & 0,97 & 0,03 & 0,94 & 0,04 & 0,99 & 0,04 \\
Nordeste & $0,59^{* * *}$ & 0,01 & $0,60^{* * *}$ & 0,02 & $0,57^{* * *}$ & 0,02 \\
Norte & $0,52^{* * *}$ & 0,01 & $0,51^{* * *}$ & 0,02 & $0,53^{* * *}$ & 0,02 \\
Centro-Oeste & $0,84^{* * *}$ & 0,02 & $0,81^{* * *}$ & 0,03 & $0,86^{* * *}$ & 0,04 \\
Sudeste & 1 & Ref & 1 & Ref & 1 & Ref \\
/cut1 & $-6,92$ & 0,08 & $-7,08$ & 0,11 & $-6,64$ & 0,11 \\
/cut2 & $-4,88$ & 0,06 & $-5,01$ & 0,08 & $-4,62$ & 0,08 \\
/cut3 & $-2,16$ & 0,05 & $-2,29$ & 0,07 & $-1,89$ & 0,07 \\
/cut4 & 1,24 & 0,05 & 1,16 & 0,07 & 1,46 & 0,07 \\
LR chi2 & $14516,12^{* * *}$ & & & \\
\hline
\end{tabular}

Fonte: Elaboração própria, com base nos microdados da Pesquisa Nacional de Saúde de 2013 - PNS.

Nota: $* * * \mathrm{p}<0,01 ; * * \mathrm{p}<0,05$. Ref: categoria de referência; NA: não se aplica; (...): informação em falta.

Em suma, pode-se destacar cinco principais diferenças entre homens e mulheres dos resultados da pesquisa:

a) As mulheres brancas tinham mais chances de declararem estado de saúde "muito bom" do que os homens brancos;

b) Mulheres que residiam na zona urbana tinham menos chances de declararem estado de saúde "muito bom" do que os homens que residiam na zona urbana;

c) Em situação de pobreza, as mulheres apresentaram mais chances que os homens de declararem estado de saúde "muito bom";

d) As mulheres que tinham mais escolaridade que o ensino médio completo, apresentaram mais chances de declararem estado de saúde "muito bom" do que os homens nas mesmas condições;

e) O número de filhos reduzia as chances de as mulheres declararem estado de saúde "muito bom". Essa variável pode estar captando parte do efeito da dupla jornada de trabalho feminina.

Considerando a abordagem da interseccionalidade, o fato de ser mulher, não branca, das áreas rurais, pobre, residir na região Nordeste e ter filho, contribui para a menor percepção de uma saúde muito boa das mulheres, tomando contornos como um importante marcador social. Por fim, as variáveis consideradas no modelo não captaram todas as diferenças de forma clara entre homens e mulheres e entre os vários grupos sociais de mulheres. Isso pode acontecer devido às características não observáveis, tais como as relações intrafamiliares que não estavam disponíveis no banco de dados. Por exemplo, as dificuldades sociais e econômicas que afetam particularmente as mulheres poderiam ser parcialmente captadas, indiretamente, caso estivesse disponível no banco de dados o número de horas dedicadas às atividades domésticas. A rotina doméstica é praticamente invisível, no entanto, a organização da casa toma muito tempo. Além disso, estaria computado dentro das atividades domésticas as tarefas de cuidarem dos filhos e de outros membros da família mais vulneráveis, por exemplo, idosos e enfermos. Todas essas tarefas tendem a ser física e emocionalmente muito desgastante, as quais perduram normalmente até a velhice dessas mulheres. 


\section{6.}

\section{CONSIDERAÇÕES FINAIS}

A percepção de boa saúde está diretamente relacionada ao bem-estar. Indivíduos saudáveis são mais produtivos, trabalham regularmente e por mais anos. Por essa razão, as decisões individuais ou políticas públicas que afetam a saúde têm impactos consideráveis sobre o desenvolvimento econômico, assim como constituem um canal para políticas de combate à desigualdade de renda e à pobreza.

O sexo ainda determina diferentes papéis, as mulheres, em média, recebem menos por hora trabalhada, ocupam cargos com menos prestígio social, estão mais propensas a condições de trabalho precário e enfrentam dupla jornada de trabalho, portanto, estão mais sujeitas ao desgaste físico e mental. As mulheres não brancas, residentes nas áreas rurais, pobres, da região Nordeste e com filho, são do grupo com menor percepção de saúde muito boa, o qual toma contorno de um importante marcador social. As diferenças de saúde se devem, em grande parte, às desigualdades nas variáveis já mencionadas e de outras não observáveis no banco de dados utilizado. As políticas de redução de desigualdade de gênero que levam em consideração a interseccionalidade de gênero na saúde podem produzir melhores resultados.

\section{REFERÊNCIAS}

ALVES, L. F.; ANDRADE, M. V.. Impactos do estado de saúde sobre os rendimentos individuais no brasil e em minas gerais. Economia Aplicada, São Paulo, v. 7, p. 359-388, 2003.

BARATA, R. B. Como e por que as desigualdades sociais fazem mal à saúde. Rio de Janeiro: Fiocruz, 2012. Disponível em: https://static.scielo.org/scielobooks/48z26/pdf/ barata-9788575413913.pdf. Acesso em: 27 abr. 2021.

BARRETO, S. M; FIGUEIREDO, R. C. Doença crônica, auto-avaliação de saúde e comportamento de risco: diferença de gênero. Revista Saúde Pública, Belo Horizonte, v. 43, n. 2, p. 38-47, 2009. Disponível em: http://www.scielo.br/pdf/rsp/v43s2/ao800.pdf. Acesso em: 27 abr. 2021.

BECKER, G. S.. Investient in human Capital: A Theoretical Analysis. Journal of Political Economy, v. 70, n. 5, p. 9-49, 1962.

BECKER, G. S. Human Capital Revisited. In: Becker G. S. Human capital: A theoretical and empirical analysis with special reference to education. 3 ed. Chicago: The University of Chicago Press; 1994. p. 15-28.

BENJAMINS, M. et al. Self-Reported health and adult mortality risk: an analysis of causespecific mortality. Social Science \& Medicine, v. 59, n. 6, p. 1297-1306, 2004. Disponível em: https:/www.sciencedirect.com/science/article/abs/pii/S0277953604000115?via\%3Dihub. Acesso em: 27 abr. 2021.

CASTRO, B. N. Percepção de saúde no Brasil: uma análise das diferenças por sexo dos trabalhadores. Dissertação (Mestrado em Economia) - Universidade Estadual do Oeste do Paraná, Toledo, 2017. Disponível em: http://tede.unioeste.br/bitstream/tede/3243/2/Bruna Castro_2017.pdf. Acesso em: 27 abr. 2021.

COSTA, R. R. F. O efeito da educação sobre estado de saúde individual no Brasil. 2008. Dissertação (Mestrado em Economia) - Centro de Desenvolvimento e Planejamento Regional da Faculdade de Ciências Econômicas da Universidade Federal de Minas Gerais, Belo Horizonte, 2008. Disponível em: http://www.dominiopublico.gov.br/pesquisa/DetalheObraForm. do?select_action=\&co_obra=167777. Acesso em: 23 jul. 2018. 
DACHS, J. N. W. Determinantes das desigualdades na auto-avaliação do estado de saúde no Brasil: análise dos dados da PNAD/1998. Ciência e Saúde Coletiva, v. 7, n. 4, p. 41-657, 2002. Disponível em: http://www.scielo.br/pdf/csc/v7n4/14596.pdf. Acesso em: 27 abr. 2021.

DEATON, A. Health, inequality, and economic development. Journal of Economic Literature, v. 41, n. 1, p. 113-158, 2003. Disponível em: https://www.princeton.edu/ deaton/downloads/ Health_Inequality_and_Economic_Development.pdf. Acesso em 27 abr. 2021.

DOYAL, L. Sex, gender, and health: the need for a new approach. BMJ Clinical, v. 323, n. 3 , p. 1061-1063, 2001. Disponível em: https://www.researchgate.net/publication/11661976_Sex_ gender_and_health_The_need_for_a_new_approach. Acesso em: 27 abr. 2021.

DWYER, D. S.; Mitchell, O. S. Health problems as determinants of retirement: Are self-rated measures endogenous? PARC Working Paper Series, Pennsylvania, v. 2, n. 98, p. 1-31, mar. 1998.

FERNANDES, A. A. et al. Envelhecimento e saúde: uma análise de gênero [relatório]. Lisboa: Faculdade de Ciências Sociais e Humana, 2012. Disponível em: https://comum.rcaap.pt/ bitstream/10400.26/6536/1/Envelhecimento\%2c\%20Sa\%c3\%bade\%20e\%20G\%c3\%a9neroRF.pdf. Acesso em: 27 abr. 2021.

FERREIRA, P. L.; SANTANA, P. Percepção de estado de saúde e de qualidade de vida da população activa: contributo para a definição de normas portuguesas. Revista Portuguesa de Saúde Pública, v. 21, n. 2, p. 15-30, 2003. Disponível em: https://estudogeral.sib. uc.pt/bitstream/10316/13656/1/Percep\%c3\%a7\%c3\%a3o\%20de\%20estado\%20de \%20 sa\%c3\%bade.pdf. Acesso em: 27 abr. 2021.

FUCHS, V. R. Reflections on the socio-economic correlates of health. Journal of Health Econonic, v. 23, n. 4, p. 653-661, 2004. Disponível em: https://www.researchgate. net/publication/8139724_Reflections_on_the_socio-economic_correlates_of_health. Acesso em: 27 abr. 2021.

GROSSMAN, M. On the concept of health capital and the demand for health. Journal of Political Economy, v. 80, n. 2, p. 223-255, 1972. Disponível em: https://pdfs.semanticscholar. org/e656/466bba4f898ad560498998639eb147f62396.pdf. Acesso em: 27 abr. 2021.

HANKIVSKY, O.; CHRISTOFFERSEN, A. Intersectionality and the determinants of health: a Canadian perspective. Critical Public Health, v. 18, n. 3, p. 271-283, 2008. Disponível em: https://www.researchgate.net/publication/244887878_Intersectionality_and_the_ Determinants_of_Health_a_Canadian_Perspective. Acesso em: 27 abr. 2021.

HOSSEINPOOR, A. R. et al. Social determinants of self-reported health in women and men: understanding the role of gender in opulation health. PLoS One, v. 7, n. 4, p. 9, 2012. Disponível em: https://www.ncbi.nlm.nih.gov/pmc/articles/PMC3326052/pdf/pone.0034799.pdf. Acesso em: 27 abr. 2021.

IDLER, E. L.; BENYAMINI, Y. Self-rated health and mortality: a review of twenty-seven community studies. Journal of Health and Social Behavior, v. 38, n. 1, p. 21-37, 1997. Disponível em: https://www.researchgate.net/publication/14118775_Self-Rated_Health_and_ Mortality_A_Review_of_Twenty-Seven_Community_Studies. Acesso em: 27 abr. 2021.

JÜRGES, H. True health vs. response styles: exploring crosscountry differences in self-reported health. Health Economics, v. 16, n. 2, p. 163-178, 2007. Disponível em: https://onlinelibrary. wiley.com/doi/pdf/10.1002/hec.1134. Acesso em: 27 abr. 2021. 
KON, A. A. Economia política do gênero: determinantes da divisão do trabalho. Revista de Economia Política, v. 22, n. 3, p. 89-109, 2002. Disponível em: http://www.rep.org.br/pdf/875.pdf. Acesso em: 27 abr. 2021.

MAIA, A. G.; RODRIGUES, C. G. Saúde e mercado de trabalho no Brasil: diferenciais entre ocupados agrícolas e não agrícolas. Revista de Economia e Sociologia Rural, v. 48, n. 4, p. 737-765, 2010. Disponível em: http://www.scielo.br/pdf/resr/v48n4/a10v48n4.pdf. Acesso em: 27 abr. 2021.

MÄKINEN, T. M.; HASSI, J. Health problems in cold work. Industrial Health, v. 47, n. 3, p. 207-220, 2009.

MELO, H. P. D. et al. Os afazeres domésticos contam. Economia e Sociedade, v. 16, n. 3, p. 435-454, 2007. Disponível em: http://www.scielo.br/pdf/ecos/v16n3/06.pdf. Acesso em: 27 abr. 2021.

NORONHA, K. V. M. D. S. A relação entre o estado de saúde e a desigualdade de renda no Brasil. Tese (Doutorado em Economia) - Universidade Federal de Minas Gerais, Belo Horizonte, 2005. Disponível em: https://repositorio.ufmg.br/handle/1843/MCCR-6W8LED Acesso em: 23 jul. 2018.

PRUS, S. G.; GEE, E. Gender differences in the influence of economic, lifestyle, and psychosocial factors on later-life health. Canadian Journal of Public Health, v. 94, n. 4, p. 1-14, 2003. Disponível em: https://www.jstor.org/stable/41993669?seq=1\#page_scan_tab_ contents. Acesso em: 27 abr. 2021.

ROSS, C.E;WU, C. The links between education and health. American SociologicalAssociation, v. 60, n. 5, p. 719-745, 1995. Disponível em: https://www.jstor.org/stable/2096319?seq=1. Acesso em: 27 abr. 2021.

SCHIEHL, A. R. et al. Qualidade de vida no trabalho e saúde. Evolução histórica e perspectivas de inovação, Belo Horizonte, v. 21, n. 1, p. 113-127, 2012.

SCHULTZ, T. W. Capital humano: investimentos em educação e pesquisa. Rio de Janeiro: Zahar Editores; 1973.

SGANZERLA, S. S. de A. Relatório e proposta de programa para grupos corporativos: atividade física na promoção da saúde e qualidade de vida. In: VILARTA, R.; GUTIERREZ, G. L. (org.). Qualidade de vida em propostas de intervenção corporativa. Campinas: IPES Editorial, 2007, p. 211-218.

SILVA, T. T. R. Qualidade de vida e promoção de saúde: uma visão estratégica para empresas. In: VILARTA, R. (org.). Qualidade de vida e políticas públicas: saúde, lazer e atividade física. Campinas: IPES Editorial, 2004. p. 133-156.

SINGH, L. et al. Determinants of gender differences in self-rated health among older population: evidence from India. SAGE Open, v. 3, n. 2, p. 1-12, 2013. Disponível em: https://journals. sagepub.com/doi/pdf/10.1177/2158244013487914. Acesso em: 27 abr. 2021.

SOUSA, E. A. Efeitos da educação sobre a saúde do indivíduo: uma análise para a região Nordeste do Brasil. Dissertação (Mestrado em Economia) - Faculdade de Economia, Administração e Contabilidade da Universidade Federal de Alagoas, Alagoas, 2010. Disponível em: http://www.feac.ufal.br/mestrado/economia/sites/default/files/dissertacoes/dissertacaoEdler.pdf. Acesso em: 27 abr. 2021. 
SOYTAS, M. A.; KOSE, T. Gender differences in self-reported health status: cross-country evidence from Turkey and the united states. Working Paper, 2014. Disponível em: https://www. econ.pitt.edu/sites/default/files/_Kose_JMP_Health_Turkey_USA_November_2014.pdf. Acesso em: 27 abr. 2021.

STIGLITZ, J. E.; SEN, A.; FITOUSSI, J. P. Report by the commission on the measurement of economic performance and social progress. CMEPSP, 2009. Disponível em: https://www. economie.gouv.fr/files/finances/presse/dossiers_de_presse/090914mesure_perf_eco_progres_ social/synthese_ang.pdf. Acesso em: 27 abr. 2021.

STOPA, Sheila Rizzato et al. Acesso e uso de serviços de saúde pela população brasileira, Pesquisa Nacional de Saúde 2013. Revista de Saúde Pública, v. 51, p. 11, 2017.

STRAUSS, J. T. D. Health, Nutrition, and Economic Development. Journal of Economic Literature, v. 36, n. 2, p. 766-817, 1998.

SZWARCWALD, C. L. et al. Determinantes da autoavaliação de saúde no Brasil e a influência dos comportamentos saudáveis: resultados da Pesquisa Nacional de Saúde, 2013. Revista Brasileira de Epidemiologia, v. 18, suppl. 2, p. 33-44, 2015. Disponível em: http://www. scielo.br/pdf/rbepid/v18s2/1980-5497-rbepid-18-s2-00033.pdf. Acesso em: 27 abr. 2021.

TAQUETTE, S. R. Interseccionalidade de gênero, classe e raça e vulnerabilidade de adolescentes negras às DST/AIDS. Saúde e Sociedade, v. 19, supll 2, p. 51-62, 2010. Disponível em: http:// www.scielo.br/pdf/sausoc/v19s2/06.pdf. Acesso em: 27 abr. 2021.

TRAVASSOS, C.; OLIVEIRA, E. X. G.; VIACAVA, F. Desigualdades geográficas e sociais no acesso aos serviços de saúde no Brasil: 1998 e 2003. Ciência \& Saúde Coletiva, v. 11, n. 2, p. 975-986, 2006.

VIEIRA-DA-SILVA, L. M.; ALMEIDA-FILHO, N. D. Eqüidade em saúde: uma análise crítica de conceitos. Cadernos de Saúde Pública, v. 25, suppl. 2, p. 217-226, 2009. Disponível em: http://www.scielo.br/pdf/csp/v25s2/04.pdf. Acesso em: 27 abr. 2021.

VINTÉM, J. M.; GUERREIRO, M. D. D., CARVALHO, H. Desigualdades de género e sociais na saúde e doença em Portugal: Uma análise do Módulo "Saúde" do European Social Survey -2004. In: Actas do VI Congresso Português de Sociologia. Lisboa, 2008. p. 1-16. Disponível em: https://cies.iscte.pt/np4EN/publicacoes?p=36688. Acesso em: 23 jul. 2018.

WEBER, L.; PARRA-MEDINA, D. Intersectionality and women's health: Charting a path to eliminating health disparities. In: Gender Perspectives on Health and Medicine. Bradford: Emerald Group Publishing Limited. 2003. Disponível https://www.researchgate. net/publication/235310386_Intersectionality_and_Women's_Health_Charting_a_Path_to Eliminating_Health_Disparities/link/5b622981a6fdccf0b206c48c/download. Acesso em: 27 abr. 2021.

WILKINSON, R. G.; MARMOT, M. (Ed.). Social determinants of health: the solid facts. World Health Organization, 2003.

WOOLDRIDGE, J. M. Econometric analysis of cross section and panel data. 2 ed. Cambridge: The MIT Press; 2010. 